\title{
Down-regulation of PSMD4 can attenuate autophagy, enhance the accumulation of intracellular ROS, and increase the sensitivity of epithelial ovarian cancer to carboplatin by inhibiting the NF- $\kappa B$ pathway
}

\author{
Ying $\operatorname{Li}^{1 \# \wedge}$, Qin Zhou ${ }^{2 \# \wedge}$, Jing Shen ${ }^{2} \wedge$, Lixia $\mathrm{Zhu}^{3} \wedge$ \\ ${ }^{1}$ Department of Gynaecology and Obstetrics, Kunshan Second People's Hospital, Kunshan, China; ${ }^{2}$ Department of Gynaecology and Obstetrics, \\ Kunshan Hospital of Traditional Chinese Medicine, Kunshan Affiliated Hospital of Nanjing University of Chinese Medicine, Kunshan, China; \\ ${ }^{3}$ Department of Gynaecology and Obstetrics, Affiliated Kunshan Hospital of Jiangsu University, Kunshan Maternal and Child Health Hospital, \\ Kunshan, China \\ Contributions: (I) Conception and design: L Zhu; (II) Administrative support: J Shen; (III) Provision of study materials or patients: Y Li, Q Zhou; (IV) \\ Collection and assembly of data: Y Li, Q Zhou; (V) Data analysis and interpretation: Q Zhou, J Shen; (VI) Manuscript writing: All authors; (VII) \\ Final approval of manuscript: All authors. \\ \#These authors contributed equally to this work. \\ Correspondence to: Lixia Zhu. Department of Gynaecology and Obstetrics, Affiliated Kunshan Hospital of Jiangsu University, Kunshan Maternal and \\ Child Health Hospital, Kunshan 215300, China. Email: doc_z1984@163.com.
}

\begin{abstract}
Background: The incidence of ovarian cancer ranks third among female gynecological cancers in the world, and more than $90 \%$ of patients are epithelial ovarian cancer (EOC). Carboplatin is the first-line chemotherapy drug for the treatment of EOC patients. However, patients who are resistant to carboplatin often do not benefit from it. Therefore, finding a key molecule that affects carboplatin sensitivity is expected to enhance the efficacy of carboplatin in EOC treatment.

Methods: The human EOC cell line SK-OV-3 and TOV-21G were used in this study. The secondgeneration sequencing technology was used to sequence the transcripts of carboplatin-resistant EOC cells and parental EOC cells. The bioinformatic analysis of the differentially expressed genes was performed by Gene Ontology (GO) enrichment, Kyoto Encyclopedia of Genes and Genomes (KEGG) pathway analysis. The EOC tissue chip in the Gene Expression Omnibus (GEO) database was also analyzed to screen the target gene. Flow cytometry, Hoechst staining, Western blot, MTS, mitochondrial specific reducing agent (Mito Tempo), and nuclear factor kappa-B (NF-кB) pathway inhibitor (BAY 11-7082) were used to explore the effect of proteasome 26S subunit, non-ATPase 4 (PSMD4) on the autophagy, apoptosis, and reactive oxygen species (ROS) accumulation of carboplatin-resistant EOC cells treated with carboplatin. In vivo, the carboplatin-resistant EOC cell lines treated with PSMD4 knockdown were injected subcutaneously into mice (twelve female BALB/c nude mice) to construct EOC subcutaneous xenograft tumor models.

Results: Based on second-generation sequencing technology and bioinformatics analysis, it was found that PSMD4 is the core molecule in the carboplatin resistance regulatory network in EOC, and its expression is up-regulated in EOC carboplatin-resistant tissues and cells. In vitro and in vivo experimental results show that the down-regulated expression of PSMD4 is closely related to the increase in sensitivity of EOC to carboplatin. Mechanically, we found that inhibiting PSMD4 expression may inhibit the activation of the NF$\kappa \mathrm{B}$ pathway, promote carboplatin-induced ROS accumulation in EOC cells, inhibit EOC cell autophagy, and enhance the sensitivity of EOC to carboplatin.
\end{abstract}

\footnotetext{
$\wedge$ ORCID: Ying Li, 0000-0002-5547-2107; Qin Zhou, 0000-0002-0919-3246; Jing Shen, 0000-0003-4168-6523; Lixia Zhu, 0000-00024982-3361.
} 
Conclusions: Down-regulation of PSMD4 may inhibit the activation of the NF- $\mathrm{B}$ pathway and autophagy, and up-regulate the level of intracellular ROS accumulation, thereby promoting carboplatin-mediated EOC cell apoptosis and enhancing carboplatin sensitivity.

Keywords: Epithelial ovarian cancer (EOC); autophagy; chemoresistance; carboplatin; nuclear factor kappa-B $(\mathrm{NF}-\kappa \mathrm{B})$

Submitted Jul 23, 2021. Accepted for publication Sep 16, 2021.

doi: $10.21037 /$ tcr-21-1389

View this article at: https://dx.doi.org/10.21037/tcr-21-1389

\section{Introduction}

Ovarian cancer is a common tumor with an incidence of about $20-30 \%$ of female genital tract malignancies (1). Global Cancer Statistics 2020 estimated that there were 295,414 new cases of ovarian cancer and 184,799 deaths from ovarian cancer (2). Studies have shown that about $70 \%$ of ovarian cancer patients are already in the advanced stage (FIGO stage III and IV) at the time of diagnosis, and the 5 -year survival rate of advanced patients is only about $29 \%$ (3).

Epithelial ovarian cancer (EOC) is the most common type of ovarian cancer, accounting for about $90 \%$ of new cases (4). At present, the standard clinical treatment plan is based on surgery supplemented with platinum-based combined chemotherapy, but the treatment effect has not been significantly improved. Currently, the first-line chemotherapy drug for EOC is carboplatin (5). Despite the initial positive response to chemotherapy, many patients gradually developed drug resistance and disease recurrence, and eventually became resistant to platinum, which made chemotherapy for EOC ineffective. Therefore, finding the key molecules that affect the sensitivity of carboplatin in EOC may help predict the therapeutic effect and better adjust the treatment plan. At the same time, this may help the development of carboplatin sensitizers or combination therapy drugs, and enhance the efficacy of carboplatin in the treatment of EOC.

Autophagy is a physiological process that is ubiquitous in normal cells and EOC cells (6). When the cells become cancerous, the level of autophagy increases under the stimulation of various pathological factors such as hypoxia, starvation, radiotherapy, and chemotherapy $(7,8)$. Its protective effect makes the cells better adapt to the harsh living environment to maintain survival, promote tumor progression, and produce multi-drug resistance. After chemotherapy drugs act on cancer cells, they induce protective autophagy and inhibit apoptosis. A study has pointed out that a certain level of autophagy persists in the EOC cell line A2780, and low-dose carboplatin can upregulate the expression of microtubule-associated protein 1 light chain 3 (LC3)-II (9).

Proteasome 26S subunit, non-ATPase 4 (PSMD4) is a regulatory subunit of the $19 \mathrm{~S}$ regulatory component of the $26 \mathrm{~S}$ proteasome (10). The PSMD4 structure contains ubiquitin-protein action motifs, which interact with ubiquitinated proteins to mediate the degradation of ubiquitinated proteins (11). In the process of tumor occurrence and development, the protein metabolism degradation pathway is often disturbed, and the intracellular homeostasis is disrupted. A previous study has found that PSMD4 may be related to the poor prognosis of patients with multiple myeloma and resistance to bortezomib, and they also found that the low expression of PSMD4 significantly enhanced the killing effect of bortezomib on tumor cells (12). However, the specific role of PSMD4 in the carboplatin sensitivity of EOC is still unclear.

In this study, based on the second-generation sequencing technology and bioinformatics methods, we first discovered that PSMD4 is the core molecule in the carboplatin resistance regulatory network of EOC, and its expression is up-regulated in carboplatin-resistant cells. In vitro and in vivo experiments have shown that the down-regulated expression of PSMD4 is closely related to the increase in carboplatin sensitivity of EOC. Further mechanism studies have found that inhibiting the expression of PSMD4 may inhibit the activation of the nuclear factor kappa-B (NF$\kappa \mathrm{B})$ pathway, thereby inhibiting autophagy, promoting carboplatin-induced reactive oxygen species (ROS) accumulation, and ultimately enhancing the sensitivity of EOC cells to carboplatin.

We present the following article in accordance with the ARRIVE reporting checklist (available at https://dx.doi. org/10.21037/tcr-21-1389). 


\section{Methods}

\section{Cell culture and transfection}

Human EOC cell lines [TOV-21G (ATCC Cat\# CRL11730, RRID:CVCL_3613), SK-OV-3 (ATCC Cat\# HTB77, RRID:CVCL_0532), TOV-112D (ATCC Cat\# CRL11731, RRID:CVCL_3612), ES-2 (ATCC Cat\# CRL-1978, RRID:CVCL_3509), and OVCAR-3 (CLS Cat\# 300307/ p690_NIH:Ovcar-3, RRID:CVCL_0465)] were purchased from the American Type Culture Collection (ATCC, Manassas, VA, USA). TOV-21G, SK-OV-3, TOV-112D, ES-2, and OVCAR-3 cells were cultured in RPMI 1640 Medium (Invitrogen, Carlsbad, CA, USA) supplemented with 10\% FBS (Invitrogen) and 1\% penicillin/streptomycin at $37{ }^{\circ} \mathrm{C}$ in $5 \% \mathrm{CO}_{2}$.

The EOC cells in the logarithmic growth phase were seeded into a six-well plate at a density of $5 \times 10^{5}$ cells/well, and cultured in a complete culture medium without antibiotics. To further study the functional role of PSMD4 in EOC cells, we established a PSMD4 silencing or overexpression model in EOC cells. A PSMD4 knockdown short hairpin RNA (shRNA) construct (shPSMD4) was purchased from Genechem (China), and the non-targeting shRNA vector was used as a negative control. The PSMD4 overexpression vector (OV-PSMD4) was purchased from Gemma Pharmaceutical Technology Co., Ltd. (China), and the empty vectors were used as a negative control. EOC cells were cultured in $60 \mathrm{~mm}$ cell culture plates and grown to $50-70 \%$ confluence and the plasmids were separately transfected into EOC cells. Transfection was conducted using Neofect DNA transfection reagent (Neofect Biotech Co., Ltd., China), which was used according to the manufacturer's protocol.

\section{Establishment of carboplatin-resistant cell lines}

Carboplatin-resistant cell lines (SK-OV-3/carboplatin and TOV-21G/carboplatin) were established by exposing SK-OV-3 and TOV-21G cells to gradually increasing the dose of carboplatin $(0.1-2.0 \mu \mathrm{g} / \mathrm{mL})$ for 5 months. Two weeks after the withdrawal of the carboplatin treatment, the biological characteristics of the resistant strains were observed. One month after the withdrawal of the carboplatin, the carboplatin-resistant cell lines can still maintain drug resistance. Compared with the half-maximal inhibitory concentration (IC50) when the carboplatin was withdrawn, there was no statistical difference in the IC50 change at 1 month after the carboplatin was withdrawn
( $P>0.05)$, indicating that the carboplatin-resistant cell lines have better drug resistance stability.

\section{Second-generation sequencing technology}

To screen for genes related to carboplatin resistance in EOC, we performed second-generation sequencing analysis on carboplatin-sensitive and resistant cell lines. The original image data file obtained by Illumina Hiseq ${ }^{\mathrm{TM}}$ is converted into the original sequencing sequence, and the quality value of the original data is statistically and visually evaluated. We used the DEGSeq or DESeq $\mathrm{R}$ function package to standardize the counts of the sample genes and calculate the multiples of gene expression differences. Then, we used the negative binomial distribution test to test the significance of the number of reads. $|\log \mathrm{FCl}|>1$ and adjust $\mathrm{P}<0.05$ are considered to be significantly differentially expressed genes, and the ggplot 2 package is used to draw a volcano map reflecting the overall distribution of differentially expressed genes.

\section{Bioinformatics analysis}

This study used Gene Expression Omnibus (GEO) database, Gene Ontology (GO), Kyoto Encyclopedia of Genes and Genomes (KEGG), STRING (https://string-db. org/) database, and DAVID (http://david.abcc.ncifcrf.gov/) database.

The differentially expressed genes related to carboplatin resistance in EOC were entered into the STRING database to search the interaction relationship between the proteins online, and the combined score $>0.4$ was used as the screening condition. The results obtained are imported into Cytoscape software for visualization processing, and a protein-protein interaction (PPI) network is constructed. Further, we used the DAVID online tool to perform GO function annotation analysis and KEGG pathway enrichment analysis on the selected differentially expressed genes and used the ClusterProfiler function package for visual display.

Finally, through the NCBI-GEO data set (https://www. ncbi.nlm.nih.gov/gds/advanced), the EOC tissue expression profile chip was searched. The GSE33482 EOC tissue chip was selected using "ovarian cancer" AND "carboplatin" as the search keywords. The chip included 21 patients with locally advanced EOC who received carboplatin-based chemotherapy. Patients were finally clinically evaluated 6 months after the end of treatment. Among them, 12 patients were in complete remission and 9 patients were 
in incomplete remission. We used the limma $\mathrm{R}$ package to analyze the differences in the expression profiles of the two groups of patients, and screened out the differential genes related to carboplatin resistance in EOC. In addition, we used the ggplot2, pheatmap, and pROC function packages to draw volcano maps, heat maps, and violin maps to verify the expression of core genes in carboplatinresistant tissues in EOC.

\section{Transmission electron microscopy}

Cells were fixed with $2.5 \%$ glutaraldehyde in phosphate buffer. The specimens were post-fixed in $1 \%$ Osmium Tetroxide with $0.1 \%$ potassium ferricyanide, dehydrated through a graded series of ethanol (30-90\%). Images were taken on a HITACHI HT7700 transmission electron microscopy system (HITACHI, Japan) at $80 \mathrm{kV}$.

\section{3-(4,5-dimethylthiazol-2-yl)-5-}

(3-carboxymethoxyphenyl)-2-(4-sulfophenyl)-2Htetrazolium (MTS) assay

Digest the cells in the logarithmic growth phase with trypsin, resuspend the culture medium, and spread a 96-well plate at a cell density of $5 \times 10^{3}$ cells/well. When the cell confluence reaches $80 \%$, different concentrations of carboplatin are added. After $24 \mathrm{~h}$, discard the drug-containing culture medium, add $20 \mu \mathrm{L}$ MTS (Shanghai Yihui Biological Technology Co., Ltd., Shanghai, China) to each well, incubate at $37^{\circ} \mathrm{C}$ in the dark for 2 to $4 \mathrm{~h}$, and detect each well with a microplate reader. The optical density (OD) was detected at a wavelength of $490 \mathrm{~nm}$. Cell survival rate \% = (average value of the experimental group - average value of background plate/average value of control group-average value of background plate) $\times 100 \%$. All experiments were repeated 3 times. The equation of IC50 of carboplatin was [viability $\%=100 /\left(1+10^{\text {[carboplatin concentration] log IC50 }}\right)$ ].

\section{Quantitative real-time PCR ( $q R T-P C R)$ analyses}

Total RNA of cell lines was extracted. GAPDH was used as the housekeeping gene. The primer sequences used for qRT-PCR were as follows: 5'-GATCGCATGCTGCCAGCCCAAC-3' (forward) and 5'-GCGCACTTCAGGCCAGCGTCAGA-3' (reverse) for PSMD4; and 5'-CTCGGCTCGACGAGCACA-3' (forward) and 5'-AACGCTGGACGACTGCCCGT-3' (reverse) for GAPDH. The relative level was calculated by the $2^{-\Delta \Delta C t}$ method.

\section{Western blot}

Total protein was extracted separately from EOC cells. A separation gel and concentrated gel were prepared, and the proteins were separated by electrophoresis and then transferred to membranes. The membranes were blocked with $5 \%$ skim milk and incubated with antibodies against PSMD4 (1:2,000, GenWay Biotech Inc. Cat\# GWB-F0AEDC, RRID:AB_10268465), LC3 (1:1,000, MBL International Cat\# PD014, RRID:AB_843283), Beclin-1 (1:1,000, US Biological Cat\# B0981-23T, RRID:AB_2064477), P62 (1:2,000, PeproTech Cat\# 500P62-50 $\mu \mathrm{g}$, RRID:AB_147647), cleaved caspase-3 (1:3,000, Cell Signaling Technology Cat\# 8202, RRID:AB_1658166), Bcl-2 (1:2,000, LSBio (LifeSpan) Cat\# LS-C438-1000, RRID:AB_1272355), Bax (1:2,000, GenWay Biotech Inc. Cat\# 18-661-15197-0.1 mg, RRID:AB_516294), NFкB p65 (1:2,000; Cell Signaling Technology Cat\# 3034, RRID:AB_330561), IkappaB-alpha (IкB $\alpha, 1: 1,000$, ABclonal Cat\# A19714, RRID:AB_2862749), Lamin B (1:2,000, LSBio (LifeSpan) Cat\# LS-B2005-50, RRID:AB_1275098), and $\beta$-actin (1:3,000, Miltenyi Biotec Cat\# 130-120-277, RRID:AB_2857517). Finally, the secondary antibody was incubated for 2 hours, and the gray band of the protein band was detected by chemiluminescence.

\section{Flow cytometry apoptosis detection}

Cell apoptosis was determined by flow cytometry after staining with Annexin V (FITC-conjugated) apoptosis kit. Briefly, EOC cells were stained with $5 \mu \mathrm{L}$ Annexin 5-FITC and propidium iodide (PI), respectively, and incubated for $15 \mathrm{~min}$ in the dark, and then analyzed by flow cytometry.

\section{Hoechst staining}

EOC cells were incubated with 5-ethynyl-20-deoxyuridine (EdU, Shanghai Huicheng Biological Technology Co., Ltd., Shanghai, China) for 5 hours. After three washes with phosphate buffer saline, cells were treated with a $1 \times$ Apollo $^{\circledR}$ reaction cocktail $(100 \mu \mathrm{L} /$ well $)$ for $30 \mathrm{~min}$. After that, each well was stained with Hoechst $33342(5 \mu \mathrm{g} / \mathrm{mL}, 100 \mu \mathrm{L} /$ well $)$ for $30 \mathrm{~min}$.

\section{Intracellular ROS detection}

First, dilute 2,7-Dichlorodi-hydrofluorescein diacetate (DCFH-DA, Beijing Biolab Technology Co., Ltd., China) 
with serum-free culture medium at 1:1,000 to make the final concentration $10 \mu \mathrm{mol} / \mathrm{L}$. After harvesting the cells in a centrifuge tube, suspend them in the diluted DCFH-DA at a cell concentration of $1 \times 10^{6}-2 \times 10^{7} / \mathrm{mL}$, and incubate in a cell culture incubator at $37^{\circ} \mathrm{C}$ for 20 minutes. During this period, mix upside down once at an interval of 3 to 5 minutes so that the cells and the probe are in full contact. Wash the cells three times with a serum-free medium to fully remove the DCFH-DA that has not entered the cells. Transfer the stained cells to a flow tube for testing.

\section{Immunobistochemistry}

Immunohistochemistry staining was performed on paraffin sections with rabbit monoclonal antibodies directed against PSMD4 (1:100; Enzo Life Sciences Cat\# PAB0242, RRID:AB_1532684), NF-кB p65 (1:100; Cell Signaling

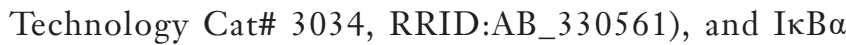
(1:100, ABclonal Cat\# A19714, RRID:AB_2862749). The biotinylated goat anti-rabbit secondary antibody (1:5,000, Signalway Cat\# L3012, RRID:AB_895483) was incubated at $4{ }^{\circ} \mathrm{C}$. Finally, the sections were incubated with $\mathrm{DAB}$ substrate for $5 \mathrm{~min}$. To validate the PSMD4, NF- $\kappa \mathrm{B}$ $\mathrm{p} 65$, and $\mathrm{I} \kappa \mathrm{B} \alpha$ antibodies used in immunohistochemistry experiments, liver cancer and para-cancerous tissue samples were used as positive and negative controls (Figure S1).

\section{In vivo tumor xenograft}

The study was conducted in accordance with the Declaration of Helsinki (as revised in 2013). Humane care was given during the experimental animal breeding and experimental procedures following the $3 \mathrm{R}$ principle of experimental animals. Experiments were performed under a project license (No. K2019001A011) granted by the Ethics Committee of the Kunshan Second People's Hospital, in compliance with the Kunshan Second People's Hospital's guidelines for the care and use of animals.

Twelve female BALB/c nude mice (20-22 g, 4-6 weeks old) were obtained from Beijing Weitong Lihua (Beijing, China). The culture of mice and subsequent experiments were carried out in SPF animal laboratories. The rearing environment of the mice is $12 \mathrm{~h}$ light and $12 \mathrm{~h}$ dark alternately, and the mice freely eat water and food. After 1 week of adaptive cultivation, follow-up experiments were carried out.

Twelve mice were divided into four groups (SK-OV-3/ carboplatin group and TOV-21G/carboplatin group) according to the random number table method, three in each group. Collect shPSMD4-SK-OV-3/carboplatin, shPSMD4-TOV-21G/carboplatin, negative control SKOV-3/carboplatin, and TOV-21G/carboplatin cells in a logarithmic growth phase. After trypsin digestion, wash with PBS solution by centrifugation, prepare cell suspension with PBS, adjust the cell density to $2 \times 10^{4}$ cells $/ \mu \mathrm{L}$ after counting, and mix with Matrigel glue at a ratio of 1:1 before use. Then, cells were subcutaneously injected into nude mice to establish the xenograft model. After the model was successfully established, carboplatin injection was used to inject the nude mice into the abdominal cavity once every 3 days at a dose of $3 \mathrm{mg} / \mathrm{kg}$ for a total of 5 times. The effect of carboplatin on transplanted tumors in mice was observed. The diameter of each xenograft tumor was measured before each administration. The tumor volume was calculated regularly according to the formula: volume of tumor $\left(\mathrm{mm}^{3}\right)$ $=\left(\right.$ length $\times$ width $\left.^{2}\right) / 2$. The nude mice were sacrificed 3 days after the last dose.

\section{Statistical analysis}

The data analysis and processing software used in this study include R version 3.6.1 (81 megabytes, 32/64 bit), Cytoscape version 3.7.2 (http://www.cytoscape.org/), GraphPad Prism version 7.0 (GraphPadSoftware, La Jolla, CA, USA). All experiments were performed in triplicate unless specified. Results were represented as the mean \pm standard deviation. The differences between normally distributed numeric variables were evaluated by Student's $t$-test. One-way analysis of variance (ANOVA) was used for the comparison among multiple groups if the variance was homogeneous. Multiple comparisons between the groups were performed using the S-N-K method. Correlations were analyzed using the Pearson correlation analysis. $\mathrm{P}<0.05$ was considered significant.

\section{Results}

Characterization of carboplatin resistance in SK-OV-3/ carboplatin and TOV-21G/carboplatin cells

Carboplatin cytotoxicity for TOV-21G, SK-OV-3, TOV112D, ES-2, and OVCAR-3 cell lines was examined. SK$\mathrm{OV}-3$ cells possessed the most sensitivity to carboplatin accompanied by the lowest IC50, whereas TOV-21G cells 
were found to be the least sensitive accompanied by the highest IC50 (Figure 1A,1B). Then, the carboplatin-resistant cell lines (SK-OV-3/carboplatin and TOV-21G/carboplatin) were established. Observing the cell morphology of each group under a light microscope, it can be seen that SKOV-3/carboplatin and TOV-21G/carboplatin cells are more slender, with binuclear cells visible, the adhesion between cells disappeared, and there are obvious intracellular blanks (Figure 1C,1D). Furthermore, we characterized carboplatin resistance in SK-OV-3/carboplatin and TOV-21G/ carboplatin cells. After exposure to gradually increasing dose of carboplatin $(0.1-2.0 \mu \mathrm{g} / \mathrm{mL})$ for 2 days, the IC50 values were $1.87 \pm 0.19 \mu \mathrm{g} / \mathrm{mL}$ and $4.89 \pm 0.54 \mu \mathrm{g} / \mathrm{mL}$ for SK-OV-3/ carboplatin and TOV-21G/carboplatin cells, respectively, showing a 4.8- and 1.8-fold increase as compared to parental cell lines (SK-OV-3 cells: $0.39 \pm 0.08 \mu \mathrm{g} / \mathrm{mL}$, Figure $1 E$; TOV$21 \mathrm{G}$ cells: $2.68 \pm 0.47 \mu \mathrm{g} / \mathrm{mL}$, Figure $1 F$ ).

\section{Screening and analysis of carboplatin resistance-related genes in EOC}

We then chose SK-OV-3 and SK-OV-3/carboplatin cell lines for sequencing analysis, and a total of 526 differentially expressed mRNAs, including 389 upregulated genes and 137 down-regulated genes, were found (Figure 2A). Subsequently, we performed GO enrichment analysis on the differentially expressed genes related to carboplatin resistance. The enrichment of the top $10 \mathrm{GO}$ projects is shown in Figure 2B. In the biological process, it is mainly concentrated in inflammation. In the cell components, it is mainly enriched in the adhesion and connection between cells. In terms of molecular function, it is mainly enriched in protein kinase activity. Then, we searched the interactions between 526 differentially expressed genes related to EOC carboplatin resistance through the STRING database and constructed a PPI network (Figure 2C). Finally, we screened the 10 differentially expressed genes (PSMD4, CCR5, NKG7, NDC80, IL21R, MZB1, OLR1, GZMH, CD3D, CXCL11) with the highest degree as core genes (Figure 2D).

We screened the array data set GSE33482 from the NCBI-GEO database for mRNAs difference analysis. This data set contains tissue samples from EOC carboplatinresistant and sensitive patients. Subsequently, the pheatmap package was used to perform a bi-clustering analysis on the 10 core genes in the PPI network, and the results are shown in Figure $3 A$. In addition, we also used the pROC package to perform receiver operating characteristic (ROC) curve analysis on 10 core genes in the PPI network. PSMD4 possesses a high prognostic value in EOC carboplatin-resistant patients [area under the curve $(\mathrm{AUC})=1.0$, Figure $3 B]$. Based on the above results, we used qRT-PCR and Western blot to detect the expression of PSMD4 in SK-OV-3, SK-OV-3/carboplatin, TOV$21 \mathrm{G}$, and TOV-21G/carboplatin cells, respectively. We found that compared with SK-OV-3 and TOV-21G cells, the mRNA and protein levels of PSMD4 in SK-OV-3/ carboplatin $(\mathrm{P}<0.01$, Figure $3 C)$ and TOV-21G/carboplatin $(\mathrm{P}<0.01$, Figure $3 D)$ cells were significantly increased. All these results are consistent with the results of our previous bioinformatics analysis.

\section{Inbibiting PSMD4 can promote the sensitivity of carboplatin-resistant EOC cells to carboplatin in vitro and in vivo}

To further verify the relationship between PSMD4 expression and EOC carboplatin sensitivity, SK-OV-3, SKOV-3/carboplatin, TOV-21G, and TOV-21G/carboplatin cells were transfected with OV-PSMD4, shPSMD4, and NC. Different concentrations of carboplatin were given to observe the changes in cell viability. QRT-PCR and Western blot results found that the expression level of PSMD4 in EOC cells transfected with shPSMD4 plasmid was significantly lower than that of the control group $(\mathrm{P}<0.01$, Figure $4 A, 4 B)$. The expression level in EOC cells transfected with PSMD4 overexpression plasmid was significantly higher than that in the control group $(\mathrm{P}<0.01$, Figure $4 C, 4 D)$. Compared with the control group, the cells in the PSMD4 low expression group were significantly more sensitive to carboplatin ( $\mathrm{P}<0.01$, Figure $4 A, 4 B$ ), while the PSMD4 overexpression group cells were significantly less sensitive to carboplatin $(\mathrm{P}<0.01$, Figure $4 C, 4 D)$. To investigate the specific function of PSMD4 in SK-OV-3/ carboplatin and TOV-21G/carboplatin cells, we achieved PSMD4 silencing by infecting cells with shPSMD4. Similarly, consistent results can be observed in the SKOV-3/carboplatin and TOV-21G/carboplatin cell lines $(\mathrm{P}<0.01$, Figure $4 E, 4 F)$.

The killing effect of carboplatin is cell apoptosis induced by DNA damage (13). Therefore, to further explore the effect of PSMD4 on carboplatin sensitivity of EOC cells, flow cytometry was performed. We found that PSMD4 knockdown enhanced cell apoptosis (Figure $5 A, 5 B$ ), 


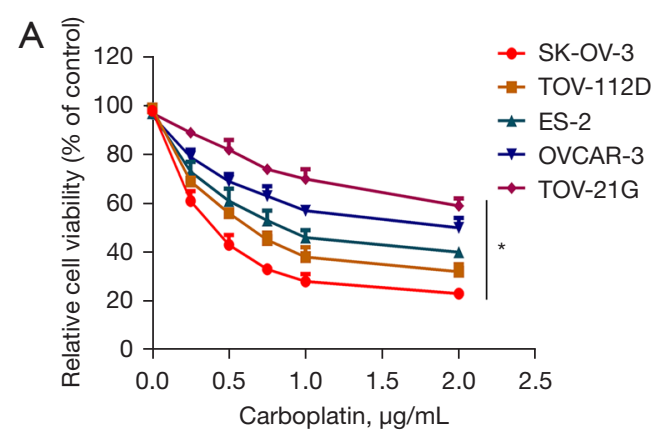

C

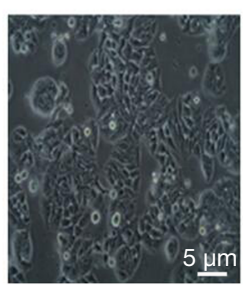

SK-OV-3

$\mathrm{E}$

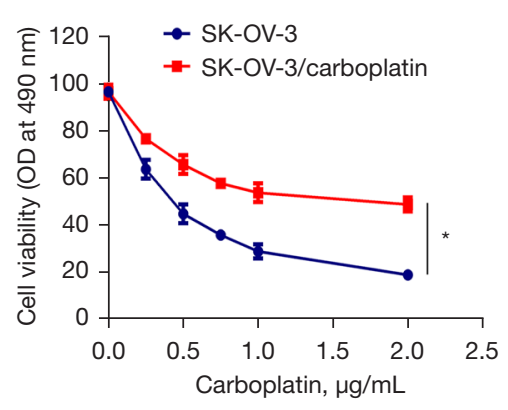

F

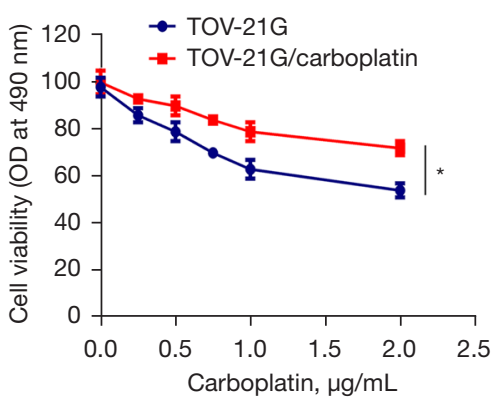

B

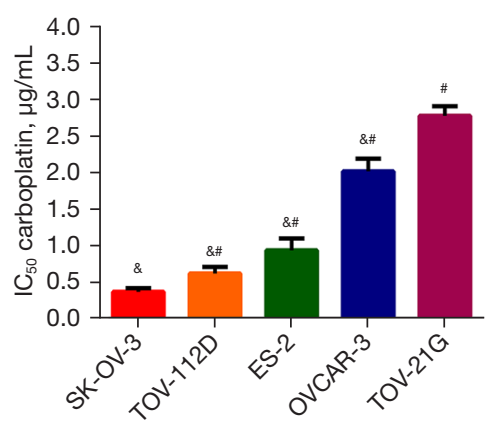

D
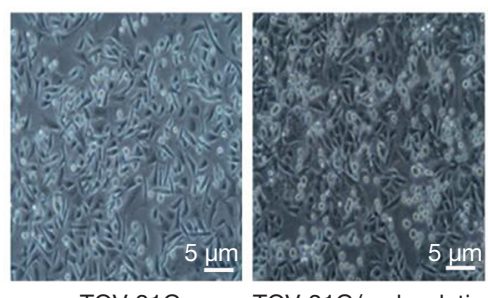

TOV-21G/carboplatin
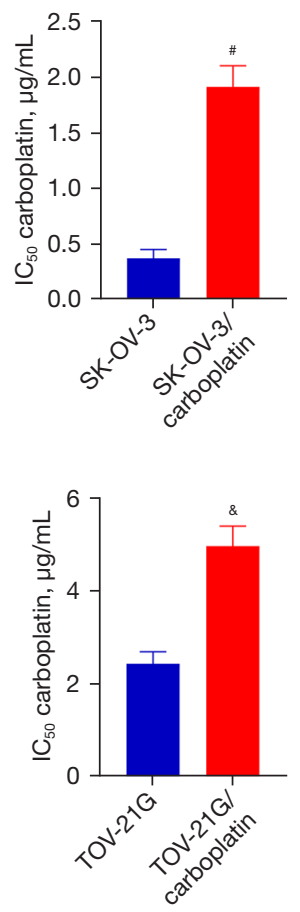

Figure 1 Characterization of carboplatin resistance in SK-OV-3/carboplatin and TOV-21G/carboplatin cells. (A,B) Carboplatin cytotoxicity for TOV-21G, SK-OV-3, TOV-112D, ES-2, and OVCAR-3 cell lines was examined. (C) Morphology of SK-OV-3 and SK-OV-3/ carboplatin cells under an optical microscope (200×). (D) Morphology of TOV-21G and TOV-21G/carboplatin cells under an optical microscope (200x). (E) The IC50 for SK-OV-3/carboplatin cells. (F) The IC50 for TOV-21G/carboplatin cells. *, one-way ANOVA was used, and $\mathrm{P}<0.05$; " , compared with SK-OV-3 group, $\mathrm{P}<0.05$; ${ }^{*}$, compared with TOV-21G group, $\mathrm{P}<0.05$. Data are expressed as mean \pm standard deviation. Scale bar $=5 \mu \mathrm{m}$. IC50, half-maximal inhibitory concentration; ANOVA, analysis of variance; OD, optical density. 

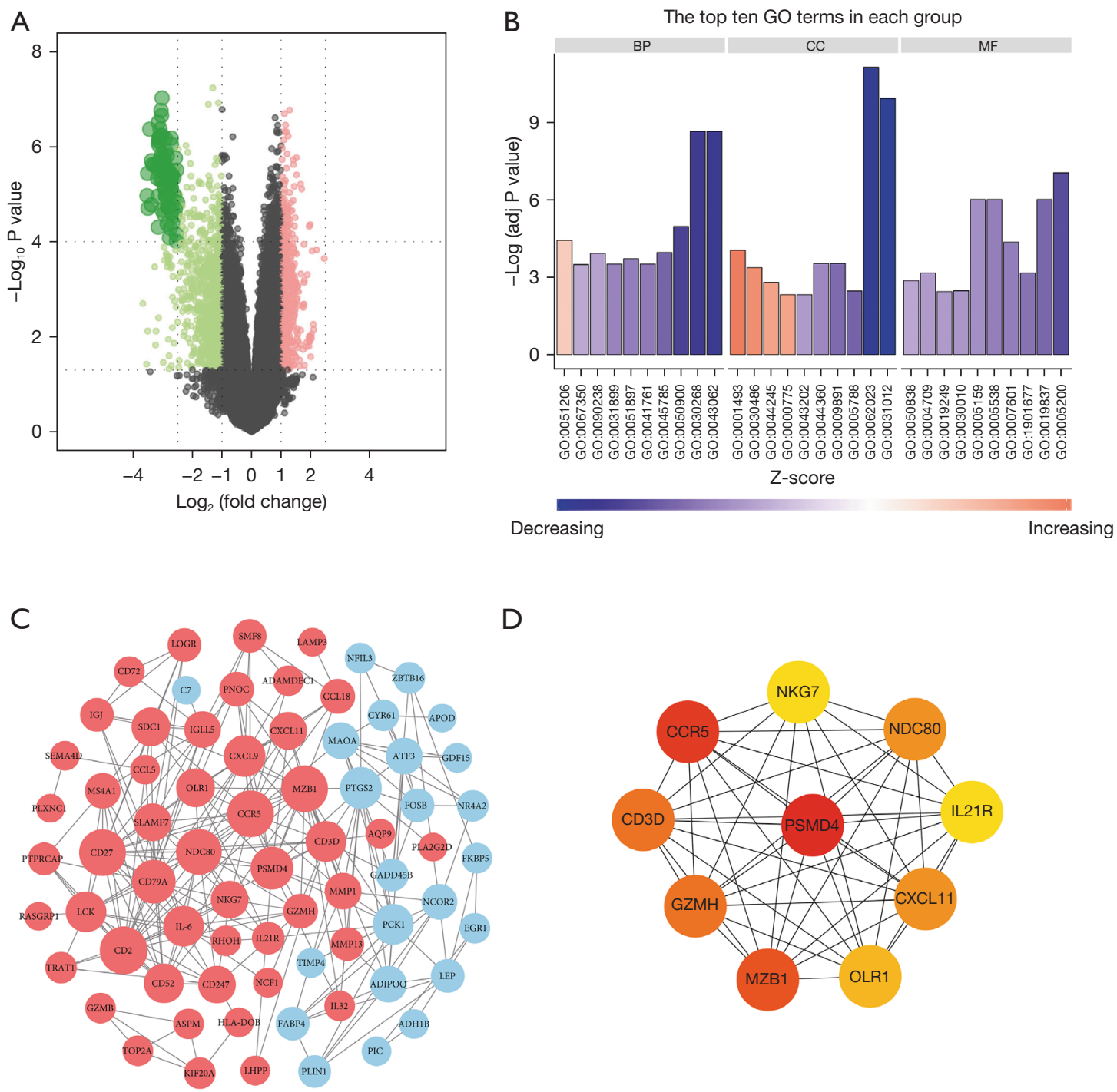

Figure 2 Volcano map, enrichment analysis, and protein interaction network analysis of differentially expressed genes related to carboplatin resistance in EOC. (A) Volcano map of differentially expressed genes related to carboplatin resistance in EOC. The red nodes represent up-regulation, and the green nodes represent down-regulation. (B) GO enrichment analysis of differentially expressed genes related to carboplatin resistance in EOC. (C) The interaction relationship between the differentially expressed genes is connected by lines. (D) The circles in the network represent the top 10 differentially expressed genes (core genes) according to a degree. The darker the color, the higher the degree. EOC, epithelial ovarian cancer; GO, Gene Ontology; BP, biological process; CC, cell components; MF, molecular function.

and nuclear fragmentation (Figure 5C) in SK-OV-3/ carboplatin and TOV-21G/carboplatin cells. Moreover, PSMD4 knockdown inhibited autophagy in SK-OV-3/ carboplatin and TOV-21G/carboplatin cells (Figure 5D). In vivo experiments further confirmed the results of cell experiments. The anti-tumor effect of carboplatin on PSMD4 down-regulated xenograft mice was significantly better than the control group, indicating that nude mice with low PSMD4 expression are more sensitive to carboplatin (Figure 5E-5G). 
A

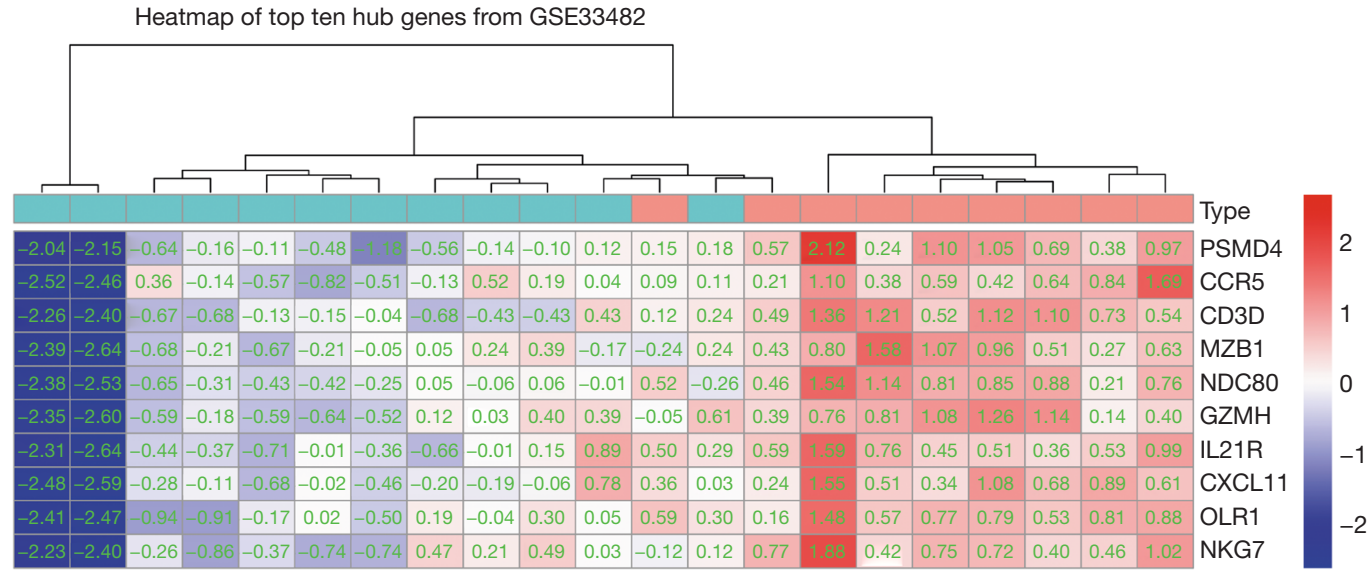

CR

NCR

B
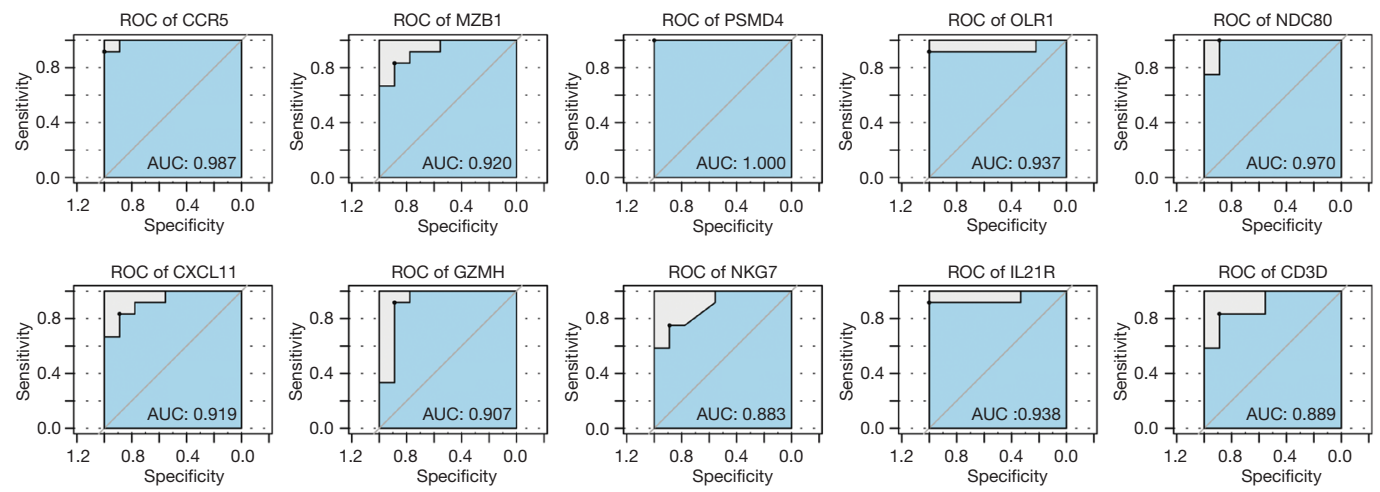

C
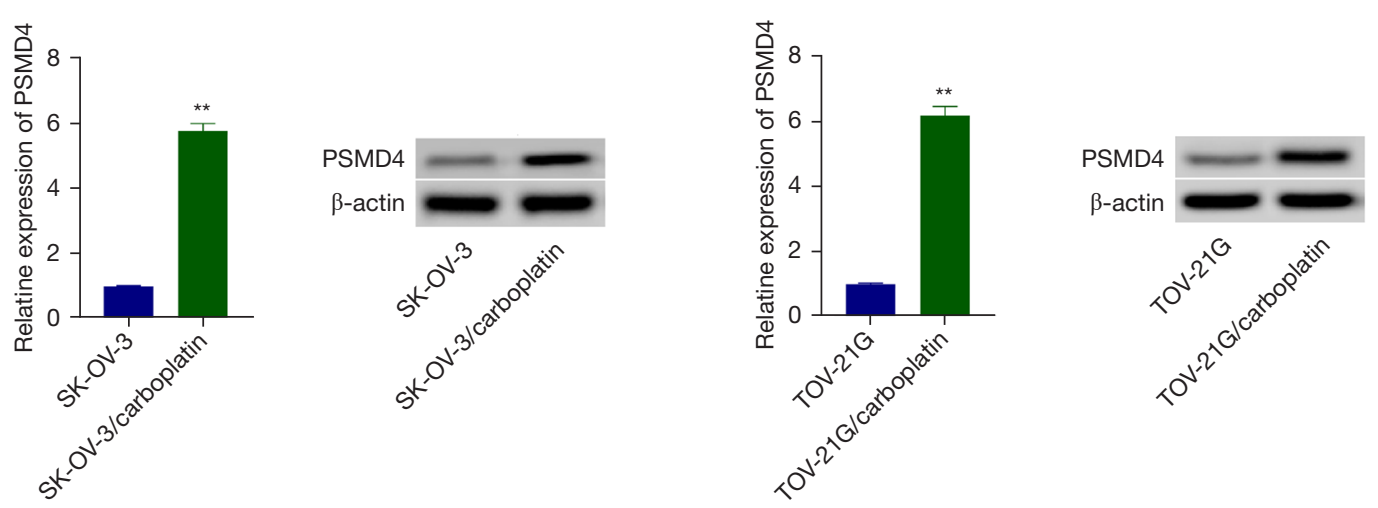

Figure 3 Comprehensive analysis of the expression of core genes in carboplatin resistance in EOC patients. (A) Double clustering analysis diagram of core genes in GSE33482 data set. (B) ROC curve analysis of core genes in carboplatin-resistant EOC patients. (C) Compared with SK-OV-3 cells, the mRNA and protein levels of PSMD4 in SK-OV-3/carboplatin cells were significantly increased. (D) Compared with TOV-21G cells, the mRNA and protein levels of PSMD4 in TOV-21G/carboplatin cells were significantly increased. Data are expressed as mean \pm standard deviation. ${ }^{* *}, \mathrm{P}<0.01$, compared to the control group. EOC, epithelial ovarian cancer; ROC, receiver operating characteristic curve; CR, complete response; NCR, non-complete response. 

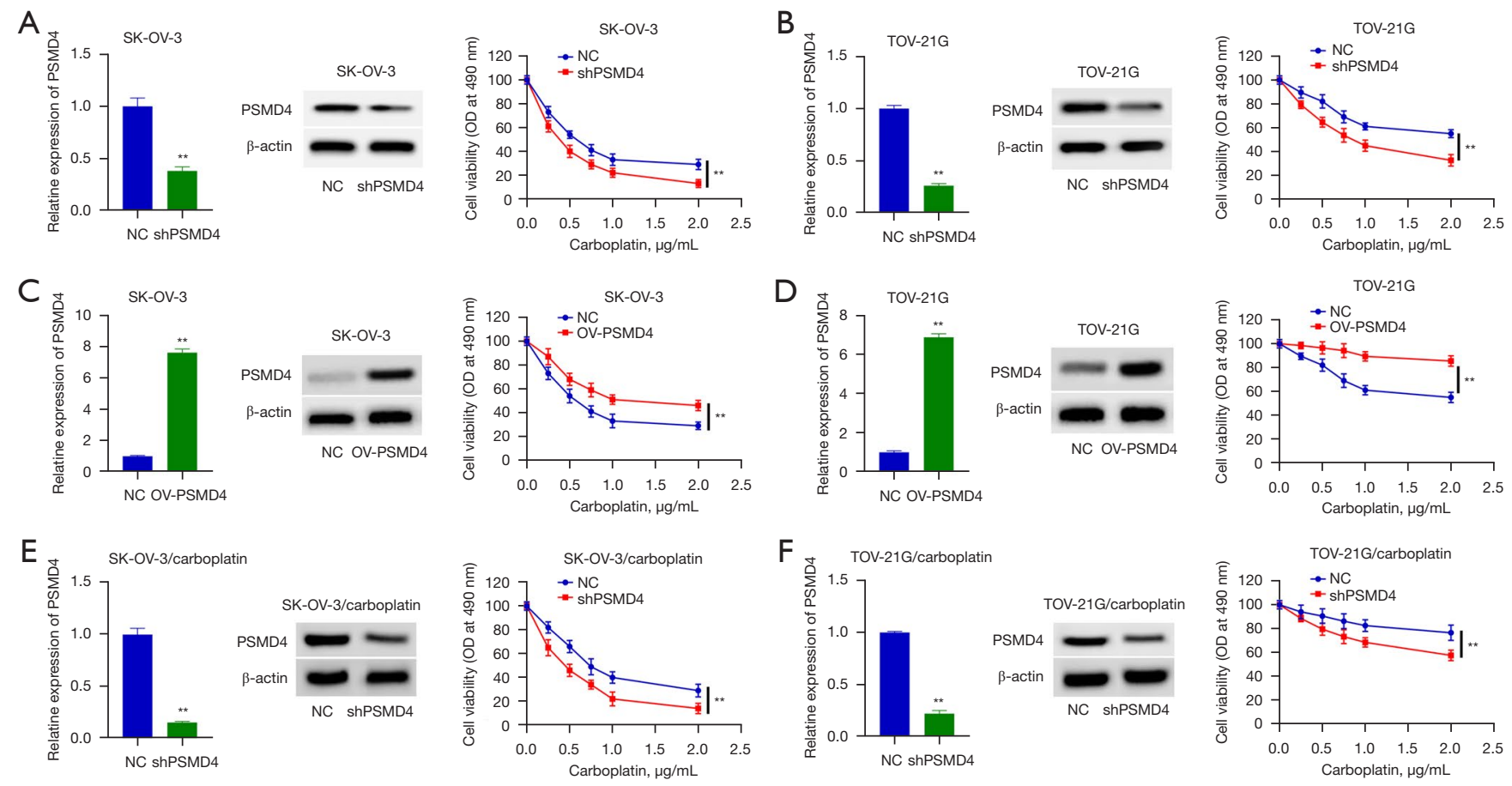

Figure 4 Construction of EOC cell models with PSMD4 overexpression and PSMD4 knockdown, and its effect on carboplatin sensitivity. (A) PSMD4 mRNA and protein expression in SK-OV-3 cells treated with shPSMD4 and changes in cell viability after 24 h of different carboplatin treatment. (B) PSMD4 mRNA and protein expression in TOV-21G cells treated with shPSMD4 and changes in cell viability after $24 \mathrm{~h}$ of different carboplatin treatment. (C) PSMD4 mRNA and protein expression in SK-OV-3 cells treated with OV-PSMD4 and changes in cell viability after $24 \mathrm{~h}$ of different carboplatin treatment. (D) PSMD4 mRNA and protein expression in TOV-21G cells treated with OV-PSMD4 and changes in cell viability after $24 \mathrm{~h}$ of different carboplatin treatment. (E) PSMD4 mRNA and protein expression in SK-OV-3/carboplatin cells treated with shPSMD4 and changes in cell viability after $24 \mathrm{~h}$ of different carboplatin treatment. (F) PSMD4 mRNA and protein expression in TOV-21G/carboplatin cells treated with shPSMD4 and changes in cell viability after 24 h of different carboplatin treatment. Data are expressed as mean \pm standard deviation. ${ }^{* *}, \mathrm{P}<0.01$, compared to the control group. EOC, epithelial ovarian cancer; PSMD4, proteasome 26S subunit, non-ATPase 4; OD, optical density.

\section{Screening and enrichment of differentially expressed genes in shPSMD4-SK-OV-3/carboplatin and NC-SK-OV-3/ carboplatin cells}

Then, we sequenced shPSMD4-SK-OV-3/carboplatin and NC-SK-OV-3/carboplatin and found a total of 869 differentially expressed mRNAs $(\mathrm{P}<0.05$ and $|\operatorname{LogFC}|>1)$, of which 254 upregulated genes and 615 down-regulated genes (Figure 6A,6B). KEGG enrichment analysis was used to explore the differentially expressed genes in shPSMD4$\mathrm{SK}-\mathrm{OV}-3 /$ carboplatin cells. The results showed that the KEGG pathway was mainly enriched in the $\mathrm{NF}-\kappa \mathrm{B}$ signaling pathway (Figure $6 C$ ). Combined with previous studies $(14,15)$, we believe that there is a close regulatory relationship between PSMD4 and NF-кB. We speculate that PSMD4 may partly mediate the NF- $\kappa \mathrm{B}$ signaling pathway and affect EOC carboplatin sensitivity.

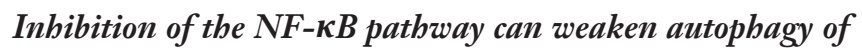
carboplatin-resistant EOC cells, increase intracellular ROS levels, and enbance carboplatin sensitivity

Studies have confirmed that carboplatin can induce the accumulation of ROS, and the accumulation of ROS is an important part of cell apoptosis $(16,17)$. Mito Tempo is a mitochondrial-targeted antioxidant that can inhibit the generation and accumulation of ROS (18). In this study, we first detected the changes in cell viability of SK-OV-3/ carboplatin and TOV-21G/carboplatin cells after the combined usage of Mito Tempo and carboplatin for $24 \mathrm{~h}$. We found that the survival rate of SK-OV-3/carboplatin 

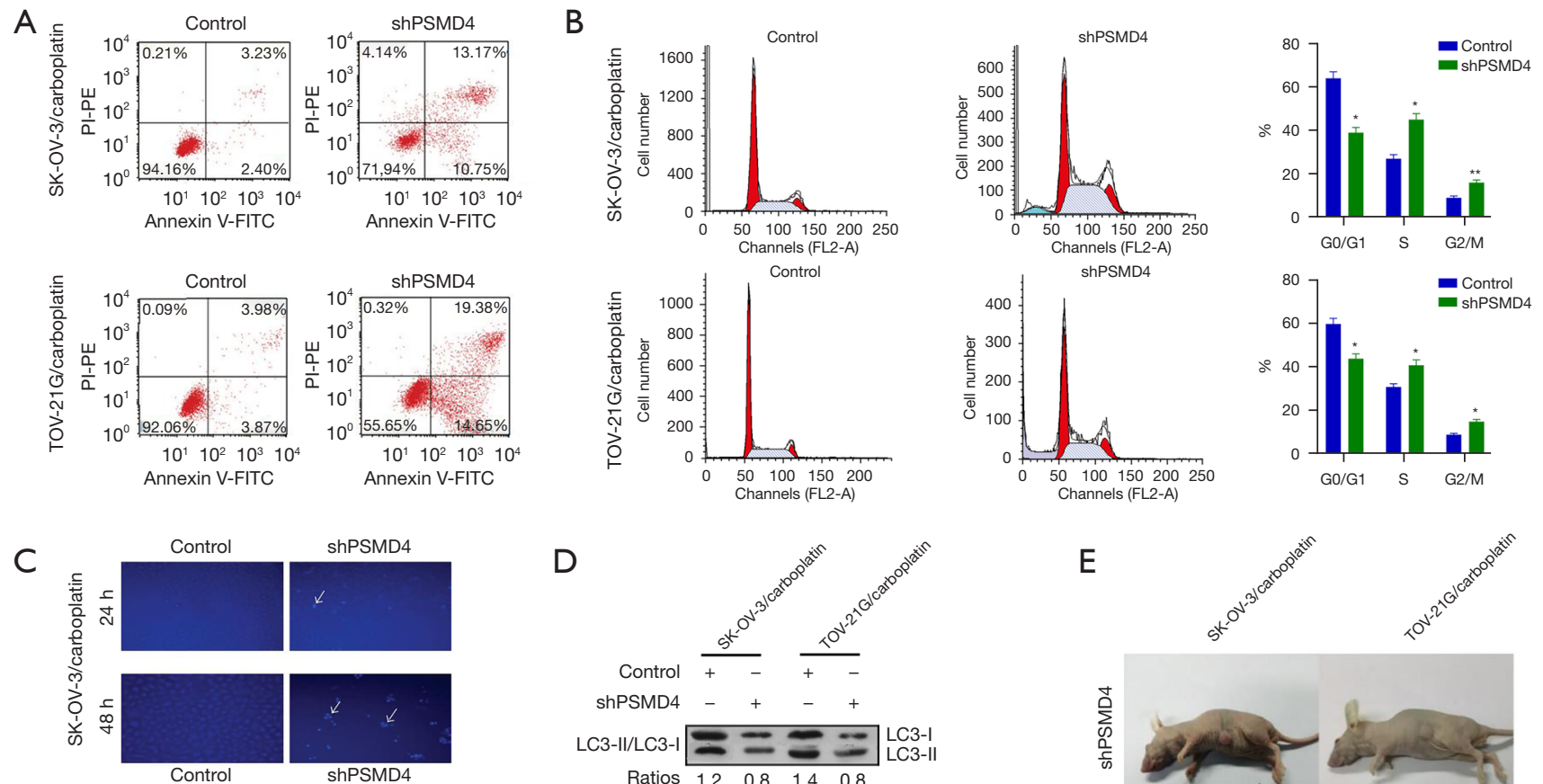

ShPSMD4

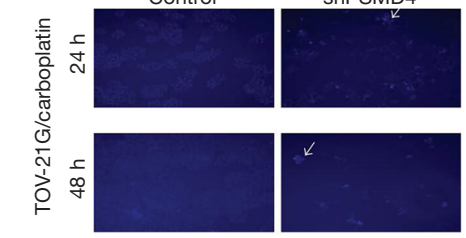

D

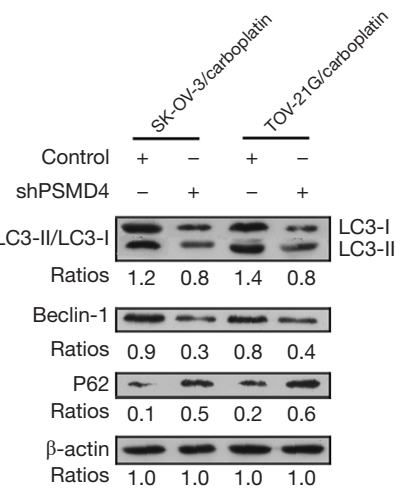

G

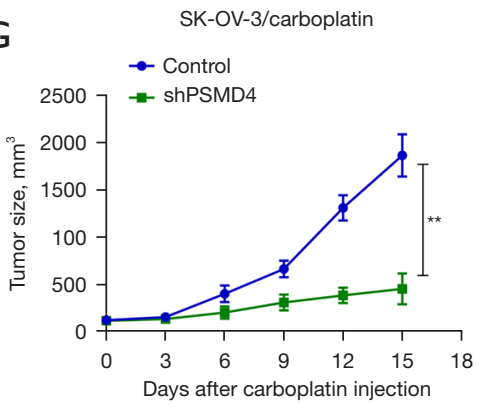

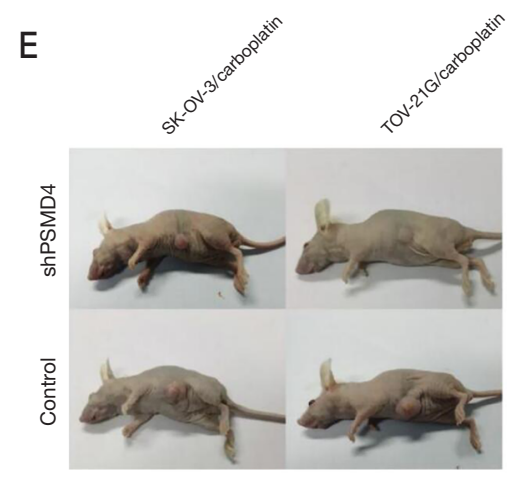

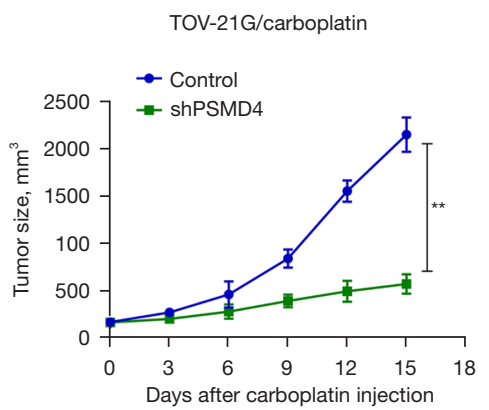

Figure 5 Inhibiting PSMD4 can promote the sensitivity of carboplatin-resistant EOC cells to carboplatin. $(A, B)$ The effect of inhibiting the expression of PSMD4 on the apoptosis of EOC cells induced by carboplatin. *, $\mathrm{P}<0.05$, compared to the control group; **, $\mathrm{P}<0.01$, compared to the control group. (C) The fluorescent dye Hoechst 33258 marks the nucleus and observes the changes of the nucleus under a fluorescence microscope (100x). Apoptotic bodies were pointed out by white arrows. (D) Effect of inhibiting PSMD4 expression on autophagy-related proteins in EOC cells induced by carboplatin. (E,F) EOC nude mouse xenograft tumor model and tumor body. (G) Changes in tumor volume of EOC nude mice after carboplatin injection. ${ }^{* *}$, one-way ANOVA was used, and $\mathrm{P}<0.01$. Data are expressed as mean \pm standard deviation. PSMD4, proteasome $26 \mathrm{~S}$ subunit, non-ATPase 4; EOC, epithelial ovarian cancer; ANOVA, analysis of variance. 


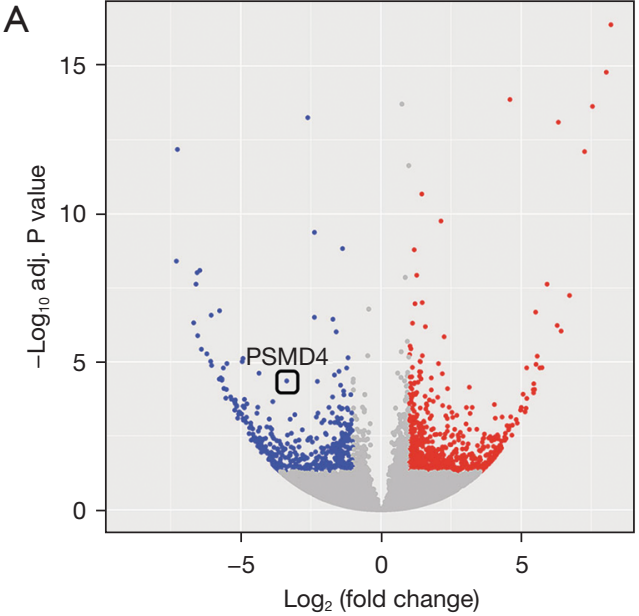

C

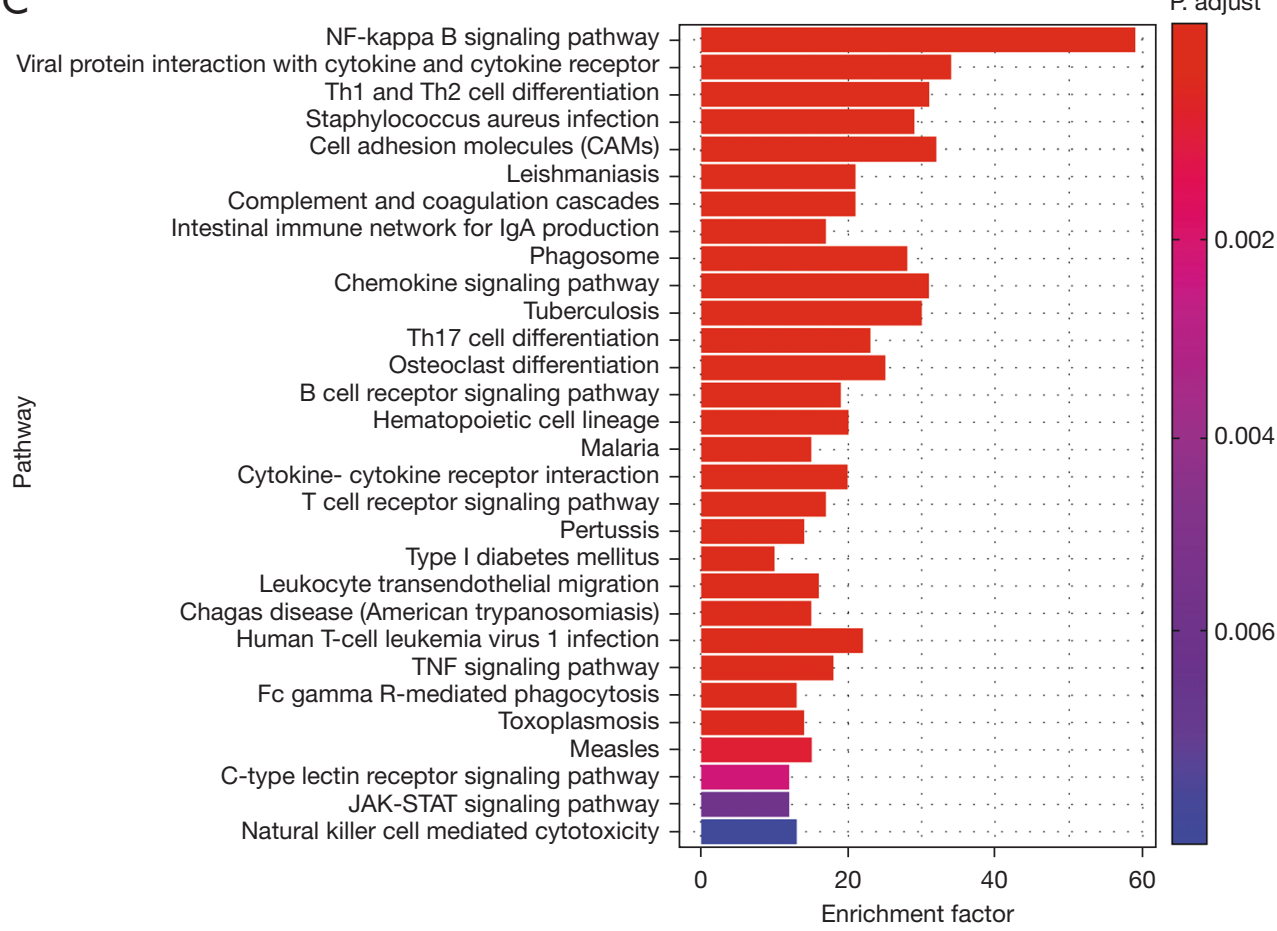

B

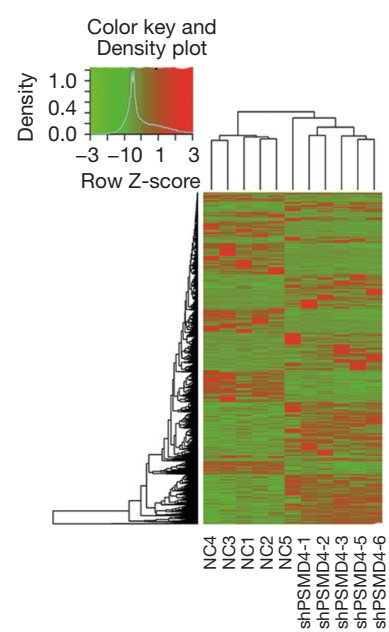

Figure 6 Screening and enrichment of differentially expressed genes in shPSMD4-SK-OV-3/carboplatin and NC-SK-OV-3/carboplatin cells. (A) Volcano map of differentially expressed genes. (B) Cluster analysis of differentially expressed genes. (C) SK-OV-3/carboplatin cells were knocked down by PSMD4 and sequenced to analyze the KEGG enrichment analysis of differentially expressed genes. PSMD4, proteasome 26S subunit, non-ATPase 4; KEGG, Kyoto Encyclopedia of Genes and Genomes.

and TOV-21G/carboplatin cells added to Mito Tempo was significantly higher than that of the control group (Figure $7 A$ ). Subsequently, we combined the NF- $\mathrm{BB}$ inhibitor (BAY 11-7082) and carboplatin to treat SK-OV-3/carboplatin and TOV-21G/carboplatin for $24 \mathrm{~h}$. MTS results showed that the survival rate of SK-OV-3/carboplatin and TOV-

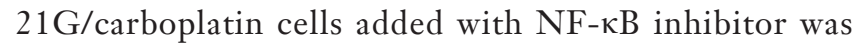
significantly lower than that of the control group (Figure $7 B$ ). Flow cytometry results also indicate that NF- $\mathrm{\kappa B}$ inhibitors can significantly up-regulate the ROS content and induce cell apoptosis under the action of carboplatin in SK-OV-3/ carboplatin and TOV-21G/carboplatin cells (Figure 7C). 

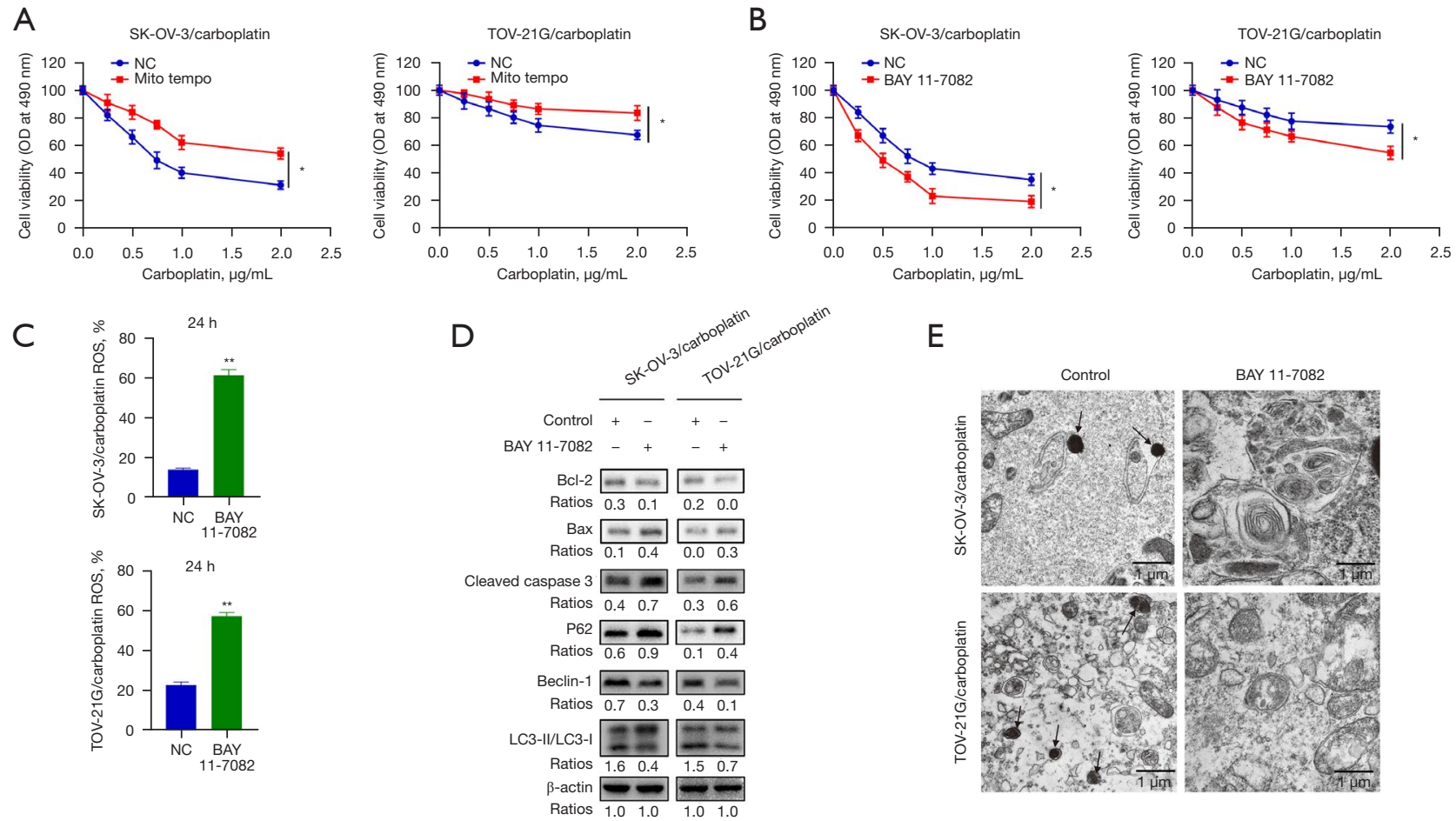

Figure 7 Inhibition of NF- $\mathrm{B}$ pathway can weaken autophagy of carboplatin-resistant EOC cells, increase intracellular ROS levels, and enhance carboplatin sensitivity. (A) MTS method was used to detect the effect of Mito Tempo on cell viability under the action of carboplatin. (B) MTS method was used to detect the effect of NF- $\mathrm{B}$ inhibitor (BAY 11-7082) on cell viability under the action of carboplatin. (C) Flow cytometry was used to detect the effect of NF- $\kappa$ B inhibitor (BAY 11-7082) on the level of ROS in carboplatinresistant EOC cells. (D) Effect of NF- $\mathrm{B}$ inhibitor (BAY 11-7082) on carboplatin-induced apoptosis and autophagy protein expression in EOC resistant cells. (E) Transmission electron microscopy was used to detect the effect of NF- $\mathrm{B}$ inhibitor (BAY 11-7082) on autophagy in carboplatin-resistant EOC cells. *, one-way ANOVA was used, and $\mathrm{P}<0.05$; **, $\mathrm{P}<0.01$, compared to the control group. Scale bar $=1 \mu \mathrm{m}$. NF-кB, nuclear factor kappa-B; ROS, reactive oxygen species; MTS, 3-(4,5-dimethylthiazol-2-yl)-5-(3-carboxymethoxyphenyl)-2(4-sulfophenyl)-2H-tetrazolium; EOC, epithelial ovarian cancer; ANOVA, analysis of variance; OD, optical density.

Western blot results showed that NF-kB inhibitors can promote apoptosis and inhibit autophagy of SK-OV-3/ carboplatin and TOV-21G/carboplatin cells (Figure 7D). Transmission electron microscopy results further confirmed the conclusion (Figure 7E).

\section{Inbibition of PSMD4 expression may enhance carboplatin sensitivity of EOC cells by mediating $N F-\kappa B$ patbway inbibition}

Subsequently, we detected the expression of NF- $\mathrm{kB}$ p65 and I $\kappa \mathrm{B} \alpha$ protein when PSMD4 was inhibited, and observed the influence of the NF- $\kappa \mathrm{B}$ pathway by detecting the expression of $\mathrm{NF}-\mathrm{\kappa B}$ p 65 protein in the cytoplasm and nucleus, respectively. The level of NF- $\kappa \mathrm{B}$ p 65 protein in the cytoplasm of shPSMD4-SK-OV-3/carboplatin and
shPSMD4-TOV-21G/carboplatin groups was significantly higher than that of the control group, while the level of NF- $\kappa \mathrm{B}$ p65 protein in the nucleus was significantly lower than that of the control group $(\mathrm{P}<0.05$, Figure $8 A)$. At the same time, the expression of I $\mathrm{KB} \alpha$ protein in shPSMD4-SKOV-3/carboplatin and shPSMD4-TOV-21G/carboplatin groups was significantly higher than that in the control group $(\mathrm{P}<0.05$, Figure $8 A)$. Since the $\mathrm{NF}-\kappa \mathrm{B}$ protein enters the nucleus to perform transcriptional regulation functions, when the NF- $\kappa \mathrm{B}$ protein entering the nucleus is significantly reduced, it indicates that the activation of the NF- $\kappa B$ pathway may be inhibited (19). At the same time, I $\mathrm{\kappa} \mathrm{B} \alpha$ protein is the regulatory protein of the NF- $\mathrm{NB}$ classical pathway, and the increase in its expression level also indirectly confirms the inhibition of NF- $\mathrm{KB}$ function (19).

To further confirm the influence of PSMD4 on the NF- 


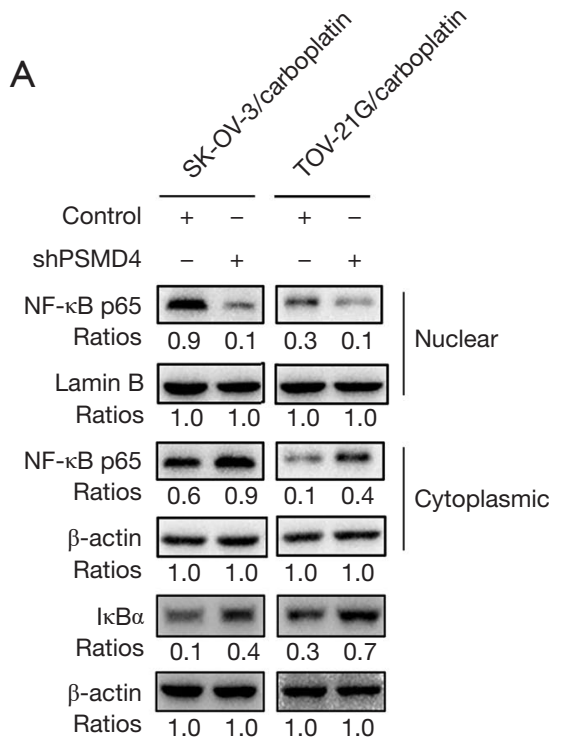

B

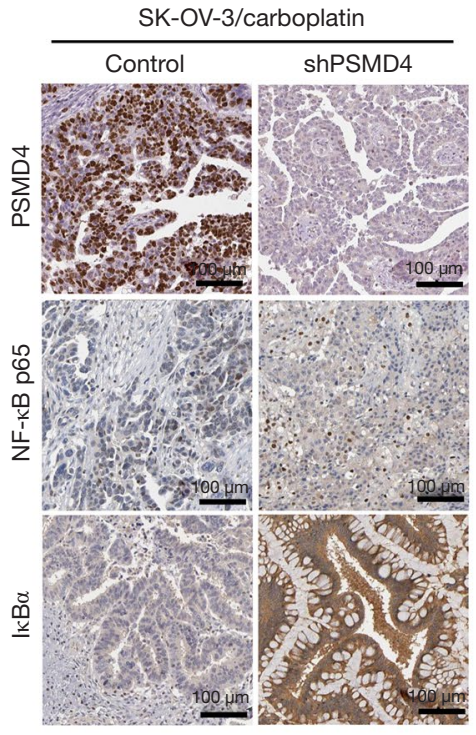

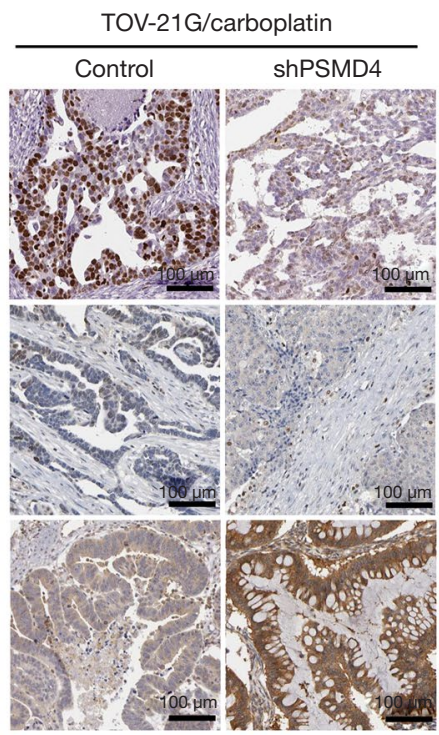

Figure 8 Inhibition of PSMD4 expression may enhance carboplatin sensitivity of EOC cells by mediating NF- $\mathrm{kB}$ pathway inhibition. (A) The effect of inhibiting the expression of PSMD4 on the NF- $\kappa \mathrm{B}$ pathway in carboplatin-resistant EOC cells. (B) Immunohistochemical

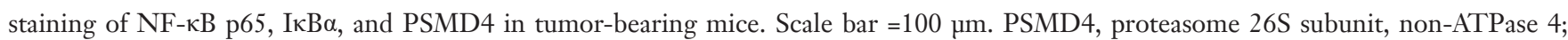

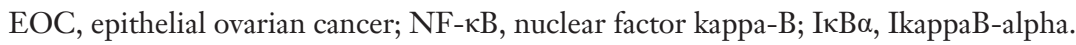

$\kappa \mathrm{B}$ pathway, we performed immunohistochemical detection of PSMD4, NF- $\kappa \mathrm{B}$ p65, and I $\kappa \mathrm{B} \alpha$ protein expression in EOC nude mice xenograft tumors. The results showed that the positive signal of PSMD4 and NF- $\kappa \mathrm{B}$ p 65 in the shPSMD4 group was lower than that of the control group, and the positive signal of $I \kappa B \alpha$ was higher than that of the control group $(\mathrm{P}<0.05$, Figure $8 B)$. The above results indicate that the down-regulation of PSMD4 may inhibit the NF- $\kappa \mathrm{B}$ p 65 and promote the IкB $\alpha$ expression.

\section{Discussion}

Ovarian cancer is one of the leading causes of cancerrelated deaths in women worldwide (2). For the treatment of ovarian cancer, surgery and radiotherapy are the first and relatively more effective treatments. However, EOC patients in some underdeveloped areas are often diagnosed at a later stage and are no longer suitable for surgery. In addition, due to a large number of patients, the waiting time for the radiotherapy machine is also very long. In this case, carboplatin-based chemotherapy is often selected. It is worth noting that for patients with advanced EOC who have recurrence and metastasis that are not suitable for local treatment, carboplatin-based systemic treatment is often used (20). Carboplatin resistance, whether it is inherent resistance or acquired resistance, will seriously affect the effectiveness of treatment. In response to this situation, we have launched a study on how to improve the sensitivity of carboplatin in EOC.

In this study, we compared SK-OV-3 and SK-OV-3/ carboplatin cell lines by transcriptome analysis and comparison. A total of 526 differentially expressed genes were screened, including 389 up-regulated differentially expressed genes and 137 down-regulated differentially expressed genes. After PPI network analysis, GEO tissue microarray data, qRT-PCR, and Western blot results, the PSMD4 protein was finally determined. PSMD4 protein contains ubiquitin protein action motifs, which can mediate the degradation of ubiquitinated proteins. In the process of protein metabolism and degradation, ubiquitin transporters are believed to promote the degradation of ubiquitin proteins (11). However, related studies have shown that the ubiquitin-proteasome system is also inhibited by ubiquitin transporters $(21,22)$. Cell experiments found that the synthesis of ubiquitin chains was inhibited by $\operatorname{Rad} 23$, and mouse experiments showed that the degradation of p53 and IкB molecules in cells was inhibited by hPLIC-2, and there was an interaction between PSMD4 and Rad23 
and hPLIC-2 $(21,22)$. A recent study has found that the interaction between the proteasome and the ubiquitinbinding protein Dsk2 is affected by PSMD4, thereby acting on the ubiquitin-proteasome degradation system (23). PSMD4 can also be hydrolyzed into small fragments and then secreted out of the cell, participating in a variety of biological processes, suggesting that the role of PSMD4 in tumors is complex (24).

To prove the relationship between PSMD4 expression and carboplatin sensitivity of EOC cells, in this study, we used MTS to detect the survival of PSMD4 low- and overexpressing cells under the action of carboplatin. It was found that the survival rate of PSMD4 down-regulated cells was significantly reduced. We then used flow cytometry and Hoechst staining to compare the apoptosis changes of carboplatin-induced cells with low PSMD4 expression and control cells. It was found that the apoptotic rate of cells with low PSMD4 expression increased significantly, and the results of Western blot also proved that the levels of cleaved caspase- 3 and Bax increased significantly, and the expression of Bcl-2 decreased significantly in cells with low PSMD4 expression. At the same time, the results of in vivo animal experiments also showed that the growing volume and rate of PSMD4 down-regulated mouse tumors after carboplatin treatment were significantly lower than those in the control group, and the sensitivity of nude mice with low PSMD4 expression to carboplatin increased. These results indicate that the inhibition of PSMD4 expression can promote carboplatin-induced EOC cell apoptosis and increase the carboplatin sensitivity of EOC cells. The positive effect of the PSMD4 in improving the chemotherapy sensitivity of EOC patients has been confirmed in some studies $(12,25,26)$. What's more, one study has found that the antiangiogenic drug cediranib can directly inhibit or inhibit the expression of the PSMD4 gene by inducing hypoxia, and then down-regulate the homologous DNA repair of tumor cells, thereby acting as a DNA repair inhibitor to enhance the sensitivity of chemotherapy drugs (10). However, it is worth noting that we constructed mouse tumor models by subcutaneous injection, which prevented us from observing the effect of down-regulation of PSMD4 on EOC metastasis. Future research can pay more attention to the effect of PSMD4 on intraperitoneal metastasis of EOC.

To further explore the mechanism of inhibiting PSMD4 in enhancing EOC carboplatin sensitivity, we sequenced shPSMD4-SK-OV-3/carboplatin and NC-SK-OV-3/ carboplatin cells using second-generation sequencing technology. The results showed that differentially expressed genes were mainly enriched in the $\mathrm{NF}-\kappa \mathrm{B}$ signaling pathway. A previous study has found that adding carboplatin to cervical cancer $\mathrm{SiHa}$ and CaSki cells can activate the NF$\kappa \mathrm{B}$ signaling pathway and induce the expression of NF$\kappa \mathrm{B}(27)$. Our Western blot results also found that in EOC carboplatin-resistant cells, the nuclear expression of NF- $\mathrm{\kappa B}$ p65 was significantly increased, and the expression of I $\mathrm{KB} \alpha$ was significantly reduced, indicating that the up-regulation of NF- $\kappa \mathrm{B}$ may inhibit EOC carboplatin sensitivity. We believe that the continuously activated $\mathrm{NF}-\mathrm{\kappa B}$ may provide a mechanism to prevent EOC cell apoptosis, which in turn leads to reduced carboplatin sensitivity.

A large number of studies have found that there are many interactions between the NF- $\kappa \mathrm{B}$ signaling pathway and ROS (28-30). The transcription of NF- $\mathrm{BB}$-dependent genes can affect the production of ROS in cells. Studies have pointed out that NF- $\kappa B$ inhibits the accumulation of ROS by inducing the expression of downstream genes FHC, thereby preventing apoptosis caused by pro-apoptotic signals (29). In this study, we used mitochondrial targeting antioxidant Mito Tempo combined with carboplatin to act on SK-OV-3/carboplatin and TOV-21G/carboplatin cells. It was found that compared with the control group, Mito Tempo can reduce carboplatin-induced apoptosis, indicating that the sensitivity of EOC cells to carboplatin may be closely related to ROS. Our inference is also supported by previous literature reports (30). At the same time, in the study, we also found that NF- $\mathrm{KB}$ inhibitors can enhance carboplatin-induced EOC drug-resistant cells SKOV-3/carboplatin and TOV-21G/carboplatin apoptosis and increase the content of intracellular ROS. Therefore, we speculate that inhibition of the NF- $\kappa \mathrm{B}$ pathway promotes the accumulation of ROS in EOC-resistant cells induced by carboplatin, thereby inducing EOC cell apoptosis.

\section{Conclusions}

In summary, the down-regulation of PSMD4 may inhibit the activation of the NF-kB pathway, inhibit autophagy, and upregulate the level of intracellular ROS accumulation, thereby promoting carboplatin-mediated EOC cell apoptosis and enhancing carboplatin sensitivity. This study may provide a new theoretical basis for the study of the mechanism of EOC carboplatin sensitivity and EOC chemotherapy. At the same time, PSMD4 and NF- $\kappa$ B pathway-related molecules may become prognostic targets for carboplatin therapy and drug resistance reversal targets for EOC patients, which will help prolong the survival of EOC patients. 


\section{Acknowledgments}

Funding: This work was supported by the Social Development Science and Technology Special Project (KS1726 to LZ).

\section{Footnote}

Reporting Checklist: The authors have completed the ARRIVE reporting checklist. Available at https://dx.doi. org/10.21037/tcr-21-1389

Data Sharing Statement: Available at https://dx.doi. org/10.21037/tcr-21-1389

Peer Review File: Available at https://dx.doi.org/10.21037/ tcr-21-1389

Conflicts of Interest: All authors have completed the ICMJE uniform disclosure form (available at https://dx.doi. org/10.21037/tcr-21-1389). The authors have no conflicts of interest to declare.

Ethical Statement: The authors are accountable for all aspects of the work in ensuring that questions related to the accuracy or integrity of any part of the work are appropriately investigated and resolved. The study was conducted in accordance with the Declaration of Helsinki (as revised in 2013). Experiments were performed under a project license (No. K2019001A011) granted by the Ethics Committee of the Kunshan Second People's Hospital, in compliance with the Kunshan Second People's Hospital's guidelines for the care and use of animals.

Open Access Statement: This is an Open Access article distributed in accordance with the Creative Commons Attribution-NonCommercial-NoDerivs 4.0 International License (CC BY-NC-ND 4.0), which permits the noncommercial replication and distribution of the article with the strict proviso that no changes or edits are made and the original work is properly cited (including links to both the formal publication through the relevant DOI and the license). See: https://creativecommons.org/licenses/by-nc-nd/4.0/.

\section{References}

1. Lyon KA, Huang JH. Bevacizumab combined with chemotherapy in platinum-resistant ovarian cancer: beyond the AURELIA trial. Transl Cancer Res 2020;9:2164-7.

2. Sung H, Ferlay J, Siegel RL, et al. Global Cancer Statistics 2020: GLOBOCAN estimates of incidence and mortality worldwide for 36 cancers in 185 Countries. CA Cancer J Clin 2021;71:209-49.

3. Palmqvist C, Staf C, Mateoiu C, et al. Increased diseasefree and relative survival in advanced ovarian cancer after centralized primary treatment. Gynecol Oncol 2020;159:409-17.

4. Amin N, Chaabouni N, George A. Genetic testing for epithelial ovarian cancer. Best Pract Res Clin Obstet Gynaecol 2020;65:125-38.

5. Alvarez Secord A, Bell Burdett K, Owzar K, et al. Predictive blood-based biomarkers in patients with epithelial ovarian cancer treated with carboplatin and paclitaxel with or without bevacizumab: results from GOG-0218. Clin Cancer Res 2020;26:1288-96.

6. Gan X, Zhu H, Jiang X, et al. CircMUC16 promotes autophagy of epithelial ovarian cancer via interaction with ATG13 and miR-199a. Mol Cancer 2020;19:45.

7. Zhao L, Kong X, Zhong W, et al. FTO accelerates ovarian cancer cell growth by promoting proliferation, inhibiting apoptosis, and activating autophagy. Pathol Res Pract 2020;216:153042.

8. Chen X, Sun Y, Wang B, et al. Prognostic significance of autophagy-related genes Beclin1 and LC3 in ovarian cancer: a meta-analysis. J Int Med Res 2020;48:300060520968299.

9. Ali JL, Lagasse BJ, Minuk AJ, et al. Differential cellular responses induced by dorsomorphin and LDN-193189 in chemotherapy-sensitive and chemotherapy-resistant human epithelial ovarian cancer cells. Int J Cancer 2015;136:E455-69.

10. AydoĞan TÜrkoĞlu S, Dayi G, KÖÇkar F. Upregulation of PSMD4 gene by hypoxia in prostate cancer cells. Turk J Biol 2020;44:275-83.

11. Fejzo MS, Anderson L, Chen HW, et al. Proteasome ubiquitin receptor PSMD4 is an amplification target in breast cancer and may predict sensitivity to PARPi. Genes Chromosomes Cancer 2017;56:589-97.

12. Shaughnessy JD Jr, Qu P, Usmani S, et al. Pharmacogenomics of bortezomib test-dosing identifies hyperexpression of proteasome genes, especially PSMD4, as novel high-risk feature in myeloma treated with Total Therapy 3. Blood 2011;118:3512-24.

13. Slootbeek PHJ, Duizer ML, van der Doelen MJ, et al. Impact of DNA damage repair defects and aggressive variant features on response to carboplatin-based 
chemotherapy in metastatic castration-resistant prostate cancer. Int J Cancer 2021;148:385-95.

14. Cheng YM, Lin PL, Wu DW, et al. PSMD4 is a novel therapeutic target in chemoresistant colorectal cancer activated by cytoplasmic localization of $\mathrm{Nrf} 2$. Oncotarget 2018;9:26342-52.

15. Lin PL, Chang JT, Wu DW, et al. Corrigendum to "Cytoplasmic localization of Nrf2 promotes colorectal cancer with more aggressive tumors via upregulation of PSMD4" Free Radic. Biol. Med. 95 (2016) 121-32. Free Radic Biol Med 2017;104:380-1.

16. Stankovic JSK, Selakovic D, Mihailovic V, et al. Antioxidant supplementation in the treatment of neurotoxicity induced by platinum-based chemotherapeutics-a review. Int J Mol Sci 2020;21:7753.

17. Mendez N, Alarcón P, Millán C, et al. Vincristine, carboplatin and cisplatin increase oxidative burst induced by PAF in canine neutrophils. Vet Immunol Immunopathol 2020;221:110011.

18. Schomberg J, Wang Z, Farhat A, et al. Luteolin inhibits melanoma growth in vitro and in vivo via regulating ECM and oncogenic pathways but not ROS. Biochem Pharmacol 2020;177:114025.

19. Li Q, Verma IM. NF-kappaB regulation in the immune system. Nat Rev Immunol 2002;2:725-34.

20. Machida H, Tokunaga H, Matsuo K, et al. Survival outcome and perioperative complication related to neoadjuvant chemotherapy with carboplatin and paclitaxel for advanced ovarian cancer: a systematic review and metaanalysis. Eur J Surg Oncol 2020;46:868-75.

21. Liang RY, Chen L, Ko BT, et al. Rad23 interaction with the proteasome is regulated by phosphorylation of its ubiquitinlike (UbL) domain. J Mol Biol 2014;426:4049-60.

Cite this article as: Li Y, Zhou Q, Shen J, Zhu L. Downregulation of PSMD4 can attenuate autophagy, enhance the accumulation of intracellular ROS, and increase the sensitivity of epithelial ovarian cancer to carboplatin by inhibiting the NF-кB pathway. Transl Cancer Res 2021;10(11):4756-4772. doi: $10.21037 /$ tcr-21-1389
22. Walters KJ, Kleijnen MF, Goh AM, et al. Structural studies of the interaction between ubiquitin family proteins and proteasome subunit S5a. Biochemistry 2002;41:1767-77.

23. Chen X, Ebelle DL, Wright BJ, et al. Structure of hRpn10 bound to UBQLN2 UBL illustrates basis for complementarity between shuttle factors and substrates at the proteasome. J Mol Biol 2019;431:939-55.

24. Cai MJ, Cui Y, Fang M, et al. Inhibition of PSMD4 blocks the tumorigenesis of hepatocellular carcinoma. Gene 2019;702:66-74.

25. Misiewicz-Krzeminska I, de Ramón C, Corchete LA, et al. Quantitative expression of Ikaros, IRF4, and PSMD10 proteins predicts survival in VRD-treated patients with multiple myeloma. Blood Adv 2020;4:6023-33.

26. Xu H, Van der Jeught K, Zhou Z, et al. Atractylenolide I enhances responsiveness to immune checkpoint blockade therapy by activating tumor antigen presentation. J Clin Invest 2021;131:146832.

27. Bravo-Cuellar A, Ortiz-Lazareno PC, Sierra-Díaz E, et al. Pentoxifylline sensitizes cisplatin-resistant human cervical cancer cells to cisplatin treatment: involvement of mitochondrial and NF-kappa B pathways. Front Oncol 2020;10:592706.

28. Ramos-Tovar E, Muriel P. Molecular mechanisms that link oxidative stress, inflammation, and fibrosis in the liver. Antioxidants (Basel) 2020;9:1279.

29. Kou X, Jing Y, Deng W, et al. Tumor necrosis factor- $\alpha$ attenuates starvation-induced apoptosis through upregulation of ferritin heavy chain in hepatocellular carcinoma cells. BMC Cancer 2013;13:438.

30. Qiu J, Zhang T, Zhu X, et al. Hyperoside induces breast cancer cells apoptosis via ROS-mediated NF- $\kappa \mathrm{B}$ signaling pathway. Int J Mol Sci 2019;21:131. 

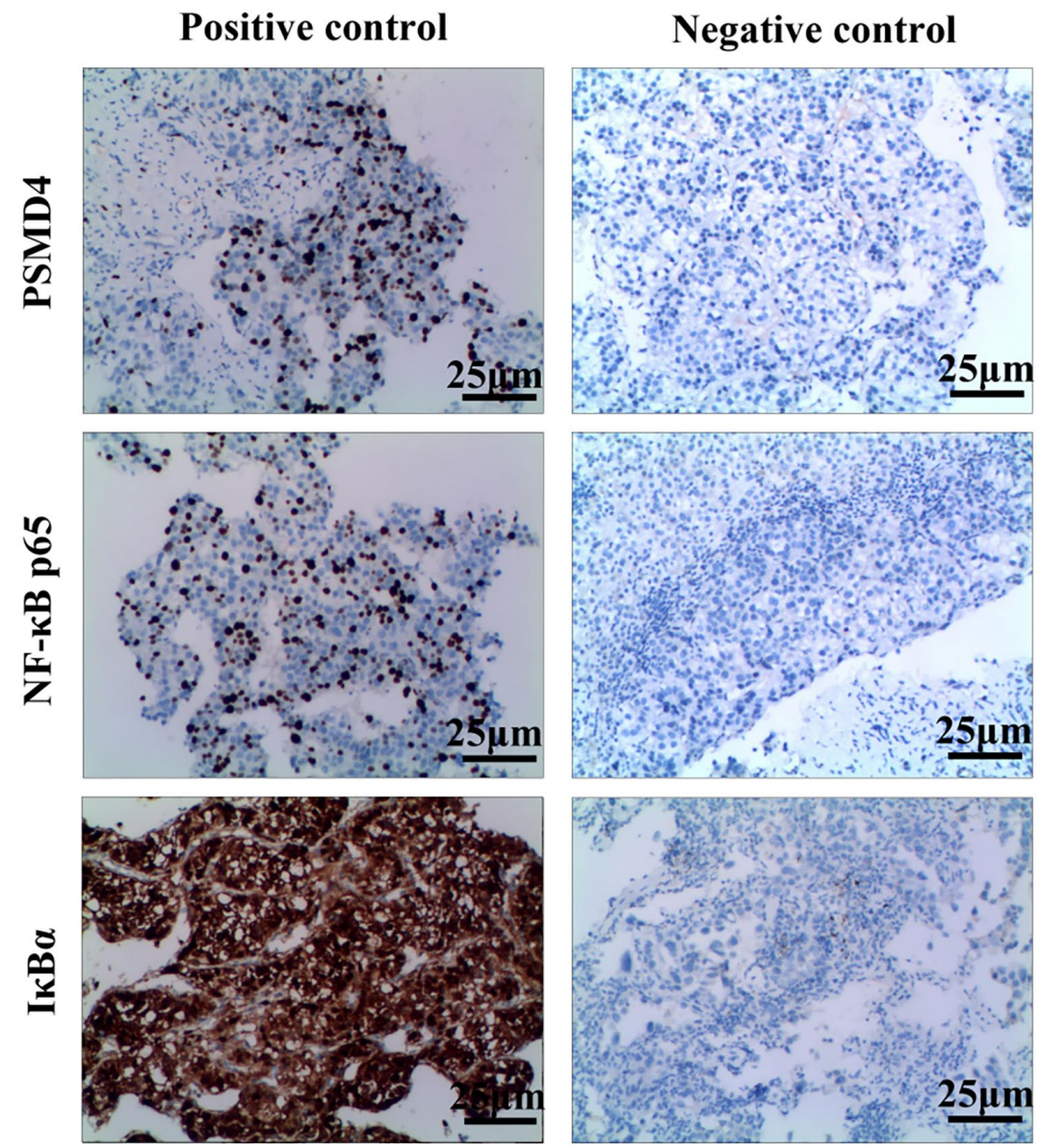

Figure S1 Liver cancer and para-cancerous tissue samples were used as positive and negative controls for the immunohistochemistry. Scale

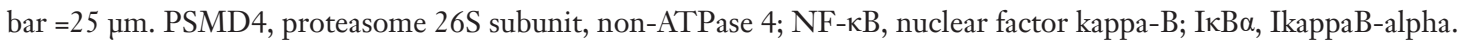

